Japan. J. Math.

Vol. 7, No. 1, 1981

\title{
A remark on the tensor product of irreducible representations of a compact Lie group
}

\author{
By C. Fronsdal and T. Hirai \\ (Received on July 2, 1980)
}

\section{$\S 1$. Introduction, and statement of the results}

Let $G$ be a connected compact Lie group with Lie algebra g. Then $G$ is necessarily reductive. Assuming here that $G$ is not abelian, we study some problems concerning the tensor product of irreducible representations of $G$, which are originally suggested by the first author's work [2]. Inspired by the Kirillov's orbital method for certain types of Lie groups, we consider orbits in $g$ under the adjoint action of $G$. (Note that $g$ can be identified with its dual space $g^{*}$ through the Killing form on [ $\left.g, g\right]$ and an appropriate inner product on the center of $g$, and the adjoint action on $g$ with the coadjoint one on $g^{*}$.) Let $T$ be a maximal torus of $G$, and $t$ its Lie algebra. Then any orbit $O$ in $g$ intersects with $t$. If $\lambda \in O \cap t$, we denote $O$ by $O(\lambda)$. Put $G(\lambda)=\{g \in G$; $\operatorname{Ad}(g) \lambda=\lambda\}$. Then $O(\lambda)$ is isomorphic to $G / G(\lambda)$, and the representation $T_{O(\lambda)}$ of $G$ constructed naturally on $L^{2}\left(O(\lambda) ; d_{\nu_{O(\lambda)}}\right)$ is equivalent to the quasi-regular representation on $L^{2}(G / G(\lambda)$; $d \bar{g})$, where $d \nu_{o(\lambda)}$ and $d \bar{g}(\bar{g}=g G(\lambda))$ denote invariant measures on $O(\lambda)$ and on $G / G(\lambda)$ respectively. On the other hand, we identify $t$ with its dual space $t *$ similarly as above, then the highest weight of an irreducible representation $U$ of $G$ is given in the form $\sqrt{-1} \mu$ with $\mu \in t$. Now our problem in its original form was to establish the following conjecture.

CONJECTURE. Let $U$ be an irreducible representation of $G$ with highest weight $\sqrt{-1} \mu$, and denote by $U^{*}$ its contragredient. Then the tensor product $U \otimes U^{*}$ is contained isomorphically in $T_{o(\lambda)}$ as a G-module, if and only if $G(\mu)$ contains a conjugate of $G(\lambda)$.

Here we define the contragredient $U^{*}$ of $U$ as follows: realize $U$ as a representation by matrices $U(g)(g \in G)$, then we put $U^{*}(g)={ }^{t} U(g)^{-1}$, where for a matrix $A,{ }^{t} A$ denotes its transposed.

Note that for any $G(\lambda)$, there exists a dominant integral form $\sqrt{-1} \lambda^{\prime}$ with

This work was done while the first author was a visiting member of the Research Institute for Fundamental Physics, Kyoto University, in the spring 1979, under the sponsorship of the Japan Society for Promotion of Sciences. 
$\lambda^{\prime} \in \mathrm{t} \cong \mathrm{t} *$ such that $G(\lambda)$ is conjugate to $G\left(\lambda^{\prime}\right)$. Further $G(\lambda)$ and $G(\mu)$ contain $T$, and are connected [3, Ch. VII, Cor. 2.8]. Let $N_{G}(T)$ be the normalizer of $T$ in $G$, then $W=N_{G}(T) / T$ is called the Weyl group of $G$. For $w \in W$, we put $G(\lambda)^{w}=g_{w} G(\lambda) g_{w}^{-1}$ and $w \lambda=\operatorname{Ad}\left(g_{w}\right) \lambda$, taking a representative $g_{w} \in N_{G}(T)$ of $w$. Then $G(\mu)$ contains a conjugate of $G(\lambda)$ if and only if $G(\mu) \supset G(\lambda)^{w}=G(w \lambda)$ for some $w \in W$. Thus the conjecture can be written as " $U \otimes U^{*} \subseteq_{T_{(\lambda)}}$ if and only if $G(\mu) \supset G(w \lambda)$ for some $w \in W$."

It is worth while remarking here that there exists a unique character $\chi_{\mu}$ of $G(\mu)$ such that $\chi_{\mu}(\exp X)=\exp (\sqrt{-1}\langle\mu, X\rangle)$ for $X \in \mathfrak{g}(\mu)$, the Lie algebra of $G(\mu)$, where $\langle\mu, X\rangle$ denotes the Killing form, and that, for a highest weight vector $v_{0}$ for $U$,

$$
U(h) v_{0}=\chi_{\mu}(h) v_{0} \quad(h \in G(\mu)) .
$$

Conversely, let $H$ be a connected subgroup of $G$ such that $U(h) v_{0} \in C v_{0}$ for $h$ $\in H$, then $H$ is contained in $G(\mu)$.

For an irreducible representation $V$ of $G$, denote by $\left[T_{o(\lambda)}: V\right]$ the multiplicity of $V$ in $T_{o(\lambda)}$. Then, by the Frobenius reciprocity theorem, we have $\left[T_{o(\lambda)}: V\right]=\left[V \mid G(\lambda): 1_{G(\lambda)}\right]$, where $1_{G(\lambda)}$ denotes the trivial representation of $G(\lambda)$. Thus " $U \otimes U^{*} G T_{o(\lambda)}$ " is equivalent to

$\left[U \otimes U^{*}: V\right] \leqslant\left[V \mid G(\lambda): 1_{G(\lambda)}\right]$ for any irreducible representation $V$ of $G$.

In case $\lambda$ is regular, we have $G(\lambda)=T$, and the conjecture says that

(1.3) for any irreducible representation $U$ of $G, U \otimes U^{*}$ is contained isomorphically in the representation $\operatorname{Ind}_{T}^{G} 1_{T}$ induced from $T$ by $1_{T}$.

In this case, we prove the following theorem. Let $w_{0}$ be the unique element in $W$ such that for any positive root $\alpha$ of $\left(\mathfrak{g}_{c}, t_{c}\right), w_{0} \alpha$ is negative (with respect to a previously fixed order).

THEOREM 1. Let $U_{i}(i=1,2)$ be irreducible representations of $G$ with highest weights $\sqrt{-1} \mu_{i}$. Then the tensor product $U_{1} \otimes U_{2}$ is contained isomorphically in $\operatorname{Ind}_{T}^{G} \chi_{\mu}$ with $\mu=\mu_{1}+w_{0} \mu_{2}$ :

$$
U_{1} \otimes U_{2} \subseteq \operatorname{Ind}_{T}^{G} \chi_{\mu_{1}}\left(\chi_{\mu_{2}}\right)^{w_{0}} \text {. }
$$

From this theorem, we get easily

Corollary. The above conjecture (1.3) is true.

In fact, let the highest weight of $U$ be $\sqrt{-1} \mu$, then that of $U^{*}$ is $-\sqrt{-1} w_{0} \mu$. Put $U_{1}=U, U_{2}=U^{*}$ in Theorem 1 , then $\chi_{\mu_{1}}\left(\chi_{\mu_{2}}\right)^{w_{0}}$ is equal to $1_{T}$ on $T$. Hence $U \otimes U^{*} \subseteq \operatorname{Ind}_{T}^{G} 1_{T}$. 
In the general case, we have not been able to get a complete answer for the conjecture. We get its "if" part from the next, theorem as explained below.

THEOREM 2. Let $U_{i}(i=1,2)$ be irreducible representations of $G$ with highest weights $\sqrt{-1} \mu_{i}$. Then, the tensor product $U_{1} \otimes U_{2}$ is contained in $\operatorname{Ind}_{H}^{G} \chi_{\mu_{1}}\left(\chi_{\mu_{2}}\right)^{w_{0}}$ with $H=G\left(\mu_{1}\right) \cap G\left(\mu_{2}\right)^{w_{0}}$ and $\left(\chi_{\mu_{2}}\right)^{w_{0}}(h)=\chi_{\mu_{2}}\left(g_{w_{0}}^{-1} h g_{w_{0}}\right)$, for $h \in$ $G\left(\mu_{2}\right)^{w_{0}}$ :

$$
U_{1} \otimes U_{2} \subseteq \operatorname{Ind}_{H}^{G} \chi_{\mu_{1}}\left(\chi_{\mu_{2}}\right)^{w_{0}}, \quad H=G\left(\mu_{1}\right) \cap G\left(\mu_{2}\right)^{w_{0}} .
$$

As a corollary of this theorem, we get

CoROLlary. Let $U$ be an irreducible representation of $G$ with highest weight $\sqrt{-1} \mu$. Then

$$
U \otimes U^{*} G_{G} \operatorname{Ind}_{G(\mu)}^{G} 1_{G(\mu)} .
$$

In fact, put $U_{1}=U, U_{2}=U^{*}$ in Theorem 2 . Then $\mu_{1}=\mu, \mu_{2}=-w_{0} \mu$ as before. Therefore $G\left(\mu_{2}\right)^{w_{0}}=G\left(w_{0} \mu_{2}\right)=G\left(-\mu_{1}\right)=G\left(\mu_{1}\right)$. Whence $H=G(\mu)$. Moreover $\chi_{\mu_{1}}\left(\chi_{\mu_{2}}\right)^{w_{0}}=1_{H}$ because $\mu_{1}+w_{0} \mu_{2}=0$. Thus we get $U \otimes U^{*} \varsigma_{\rightarrow} \operatorname{Ind}_{H}^{G} 1_{H}$ with $H=G(\mu)$.

From this corollary, we get the "if" part of the conjecture.

Proposition. Let $U$ be an irreducible representation of $G$ with highest weight $\sqrt{-1} \mu$. If $G(\mu)$ contains a conjugate of $G(\lambda)$, then the tensor product $U \otimes U^{*}$ is contained isomorphically in $\operatorname{Ind}_{G(\lambda)}^{G} 1_{G(\lambda)}$.

In fact, let $H^{\prime}$ be a conjugate of $G(\lambda)$ contained in $H=G(\mu)$. Then $\operatorname{Ind}_{H^{\prime}}^{G} 1_{H^{\prime}}$ is equivalent to $\operatorname{Ind}_{G(\lambda)}^{G} 1_{G(\lambda)}$, and contains isomorphically $\operatorname{Ind} d_{H}^{G} 1_{H}$.

We must remark that we take here the following definition of the induced representation.

Definition. Let $H$ be a closed subgroup of $G$, and $\chi$ a character of $H$. Then $\operatorname{Ind}_{H}^{G} \chi$ is realized by right translations on the space of functions $\{f \in$ $\left.L^{2}(G) ; f(h g)=\chi(h) f(g)(h \in H, g \in G)\right\}$.

We note that Theorem 2 can also be stated in the following form. For this interpretation, we are indebted to Prof. N. Tatsuuma.

Theorem 3. Let the notations be as in Theorem 2. Then the tensor product $U_{1} \otimes U_{2}$ is always cyclic and has a cyclic vector $v$ satisfying

$$
\left(U_{1} \otimes U_{2}\right)(h) v=\left(\chi_{\mu_{1}}\left(\chi_{\mu_{2}}\right)^{w_{0}}\right)(h) v \quad\left(h \in H=G\left(\mu_{1}\right) \cap G\left(\mu_{2}\right)^{w_{0}}\right) .
$$

In particular, $U_{1} \otimes U_{1}^{*}$ has a cyclic vector invariant under $G\left(\mu_{1}\right)$. 
In fact, similarly as (1.2), the inclusion relation in Theorem 2 is interpreted as

" $\left[U_{1} \otimes U_{2}: V\right] \leqslant\left[V \mid H ; \chi_{\mu_{1}}\left(\chi_{\mu_{2}}\right)^{w_{0}}\right]$ for every irreducible representation $V$."

This in turn is equivalent to the assertion in Theorem 3.

Now, before explaining the contents of the subsequent sections, we remark that Theorem 1 is a consequence of Theorem 2 . In fact, $H$ in Theorem 2 contains $T$, and the restriction on $T$ of the character $\chi_{\mu_{1}}\left(\chi_{\mu_{2}}\right)^{w_{0}}$ of $H$ is the one denoted by the same symbol in Theorem 1 .

In the next section, $\S 2$, we prove Theorem 2 . In $\S 3$, we give certain inequalities for multiplicities and for the Kostant's partition function (Theorem 4) by interpreting the inclusion relation in Theorem 1. In the last section, $\S 4$, we give some examples for $G=U(n)$ which support the "only if" part of the conjecture, and we see that it is true for $U(3)$.

\section{§. Proof of Theorem 2}

To prove Theorem 2, we may assume that $G$ is semisimple. Further, if $G$ is semisimple, replacing it by its universal covering group if necessary, we may also assume that $G$ is simply connected. Assume them from now on.

Let $g_{c}$ and $t_{c}$ be the complexification of $g$ and $t$ respectively. We fix an order in the set of roots of $\left(\mathfrak{g}_{c}, \mathfrak{t}_{c}\right)$. Let $\mathfrak{n}$ be a maximal nilpotent subalgebra of $\mathfrak{g}_{c}$ spanned by the root subspaces $\mathrm{g}^{\alpha}$ with $\alpha<0$, and put $\mathfrak{b}=\mathfrak{t}_{c}+\mathfrak{n}$, a Borel subalgebra. Let $G_{c}$ be a simply connected Lie group with Lie algebra $g_{c}$, then the analytic subgroup corresponding to $g$ is naturally isomorphic to $G$. We identify them. Let $B$ and $N$ be the analytic subgroups corresponding to $\mathfrak{b}$ and $\mathfrak{n}$ respectively.

Let $\sqrt{-1} \mu$ with $\mu \in \mathrm{t} \cong t^{*}$ be a dominant integral form. Then the complexification $g_{c}(\mu)$ of $g(\mu)$ is spanned by $t_{c}$ and $g^{\alpha}$ with $\alpha$ satisfying $s_{\alpha} \mu=\mu$, where $s_{\alpha} \in W$ denotes the reflexion corresponding to $\alpha$. Let $G_{c}(\mu)$ be the analytic subgroup of $G_{c}$ corresponding to $g_{c}(\mu)$, and put $P(\mu)=G_{c}(\mu) N$. Then, since $\mu$ is dominant, $P(\mu)$ is a parabolic subgroup of $G_{c}$ containing $B$, which corresponds canonically to the set of simple roots $\alpha$ such that $s_{\alpha} \mu=\mu$.

To prove Theorem 2, we use the following two facts.

(1) Every irreducible representation of $G$ can be extended to a holomorphic representation of $G_{c}$.

(2) The subset $B w_{0} B$ contains a non-void open subset of $G_{c}$.

Note 1. In reality, $B w_{0} B$ is itself open and dense in $G_{c}$. This can be seen from the Bruhat decomposition of $G_{c}$ into $B$-double cosets and the closure relation among them. However, (2) is proved easily as follows. Put 
$N_{+}=w_{0} N w_{0}^{-1}$, then it is a subgroup corresponding to the nilpotent subalgebra spanned by $\mathrm{g}^{\alpha}$ 's with $\alpha>0$. Let $T_{c}$ be the Cartan subgroup of $G_{c}$ corresponding to $t_{c}$. Then $B w_{0} B w_{0}{ }^{-1}=N T_{c} N_{+}$. From this, (2) follows.

Let $U$ be an irreducible representation of $G$ with highest weight $\sqrt{-1} \mu$ on a vector space $E$. Denote again by $U$ the holomorphically extended representation of $G_{c}$ from $U$, and also again by $\chi_{\mu}$ the holomorphically extended character of $G_{c}(\mu)$ from $\chi_{\mu}$. Let $E^{*}$ be the dual space of $E$. Then, for $v \in E$, $v^{*} \in E^{*}$

$$
\left(U(g) v, v^{*}\right)=\left(v, U^{*}\left(g^{-1}\right) v^{*}\right) \quad\left(g \in G_{c}\right),
$$

where $(\cdot, \cdot)$ denotes the pairing of $E$ and $E^{*}$. Let $v_{0}^{*} \neq 0$ be a lowest weight vector for $U^{*}$. The lowest weight of $U^{*}$ is $-\sqrt{-1} \mu$. Therefore

$$
U^{*}(h) v_{0}^{*}=\chi_{\mu}(h)^{-1} v_{0}^{*} \quad\left(h \in G_{c}(\mu)\right), \quad U^{*}(n) v_{0}^{*}=v_{0}^{*} \quad(n \in N) .
$$

Consider the space $D_{\mu}$ of holomorphic functions on $G_{c}$ spanned by

$$
f_{v}(g)=\left(v, U^{*}\left(g^{-1}\right) v_{0}^{*}\right)=\left(U(g) v, v_{0}^{*}\right) \quad\left(g \in G_{c}\right),
$$

for $v \in E$. Then for $f \in D_{\mu}$,

$$
f(p g)=\chi_{\mu}(p) f(g) \quad(p \in P(\mu)),
$$

where $\chi_{\mu}$ denotes the character of $P(\mu)$ extended from $G_{c}(\mu)$ by putting $\chi_{\mu}(n)$ $=1$ for $n \in N$. The space $E$ is isomorphic to $D_{\mu}$, and the representation $U$ on $E$ is transformed to $\tilde{U}$ on $D_{\mu}$ as

$$
\tilde{U}\left(g_{0}\right) f(g)=f\left(g g_{0}\right) \quad\left(g_{0}, g \in G_{c}\right) .
$$

Thus we get the following

LEMMA 2.1. Let $U$ be an irreducible representation of $G$ with highest weight $\sqrt{-1} \mu$. Then the holomorphically extended one is contained in the representation by right translations on the space of holomorphic functions on $G$ satisfying (2.3).

Now let $U_{1}, U_{2}$ be as in Theorem 2. Consider the tensor product $\tilde{U}_{1} \otimes \tilde{U}_{2}$ of the representations $\tilde{U}_{1}$ and $\tilde{U}_{2}$ of $G_{c}$. It is given on the space $D=D_{\mu_{1}} \otimes D_{\mu_{2}}$ as follows: for $\varphi \in D$,

$$
\left(\tilde{U}_{1} \otimes \tilde{U}_{2}\right)\left(g_{\theta}\right) \varphi\left(g_{1}, g_{2}\right)=\varphi\left(g_{1} g_{0}, g_{2} g_{0}\right) \quad\left(g_{0} \in G_{c}\right) .
$$

On the other hand, $\varphi \in D$ is a holomorphic function on $G_{c} \times G_{c}$ satisfying

$$
\varphi\left(p_{1} g_{1}, p_{2} g_{2}\right)=\chi_{\mu_{1}}\left(p_{1}\right) \chi_{\mu_{2}}\left(p_{2}\right) \varphi\left(g_{1}, g_{2}\right) \quad\left(g_{1}, g_{2} \in G_{c}, p_{1} \in P\left(\mu_{1}\right), p_{2} \in P\left(\mu_{2}\right)\right) .
$$


Let $\Delta$ be the diagonal $\left\{(g, g) ; g \in G_{c}\right\}$ in $G_{c} \times G_{c}$, then $\Delta \cong G_{c}$. Consider the double coset $\Omega=\left(P\left(\mu_{1}\right) \times P\left(\mu_{2}\right)\right)\left(1, w_{0}\right) \Delta$ in $G_{c} \times G_{c}$, where 1 and $w_{0}$ in $W$ must be replaced by their representatives in $N_{G}(T)$. Then we see from (2) that $\Omega$ contains a non-void open subset. Therefore the map $\varphi \mapsto \varphi_{\Omega}=\left.\varphi\right|_{\Omega}$ of $D$ is $1-1$, and it follows from (2.6) that

$$
\varphi_{\Omega}\left(p_{1} g, p_{2} g_{w_{0}} g\right)=\chi_{\mu_{1}}\left(p_{1}\right) \chi_{\mu_{2}}\left(p_{2}\right) \varphi_{\Omega}\left(g, g_{w_{0}} g\right) \quad\left(g \in G_{c}, p_{1} \in P\left(\mu_{1}\right), p_{2} \in P\left(\mu_{2}\right)\right) .
$$

Put $\psi(g)=\varphi_{\Omega}\left(g, g_{w_{0}} g\right)=\varphi\left(g, g_{w_{0}} g\right)$, then $\psi$ is holomorphic on $G_{c}$. Note that $g_{w_{0}} p g_{w_{0}}^{-1} \in P\left(\mu_{2}\right)$ is equivalent to $p \in g_{w_{0}}^{-1} P\left(\mu_{2}\right) g_{w_{0}}=P\left(\mu_{2}\right)^{w_{0}}=G_{c}\left(\mu_{2}\right)^{w_{0}} N^{+}$because $w_{0}^{2}=1$. Since $P\left(\mu_{1}\right) \cap P\left(\mu_{2}\right)^{w_{0}} \supset G_{c}\left(\mu_{1}\right) \cap G_{c}\left(\mu_{2}\right)^{w_{0}}$, we get from (2.7)

$$
\psi(h g)=\chi_{\mu_{1}}(h)\left(\chi_{\mu_{2}}\right)^{w_{0}}(h) \psi(g) \quad\left(g \in G_{c}, h \in G_{c}\left(\mu_{1}\right) \cap G_{c}\left(\mu_{2}\right)^{w_{0}}\right) .
$$

Put $f=\psi \mid G$. Since $\psi$ is holomorphic, the map $\psi \mapsto f$ is $1-1$. Moreover, since $G \cap G_{c}\left(\mu_{1}\right) \cap G_{c}\left(\mu_{2}\right)^{w_{0}} \supset G\left(\mu_{1}\right) \cap G\left(\mu_{2}\right)^{w_{0}}, f$ satisfies that

$$
f(h g)=\chi_{\mu_{1}}(h)\left(\chi_{\mu_{2}}\right)^{w_{0}}(h) f(g) \quad\left(g \in G_{c}, h \in G\left(\mu_{1}\right) \cap G\left(\mu_{2}\right)^{w_{0}}\right) .
$$

Thus the image $\mathscr{E}$ of the space $D$ under $\varphi \mapsto \psi \mapsto f$ is a subspace of continuous functions on $G$ satisfying (2.9). The representation $\tilde{U}_{1} \otimes \tilde{U}_{2}$ of $G_{c}$ on $D$ is transformed after the restriction on $G$ into that on $\mathscr{E}$ by right translations.

This proves that the tensor product $U_{1} \otimes U_{2}$ is contained isomorphically in $\operatorname{Ind}_{H}^{G} \chi_{\mu_{1}}\left(\chi_{\mu_{2}}\right)^{w_{0}}$ with $H=G\left(\mu_{1}\right) \cap G\left(\mu_{2}\right)^{w_{0}}$. Thus the proof of Theorem 2 is now complete.

Note 2. Theorem 1 asserts that $U \otimes U^{*} \subseteq \operatorname{Ind}_{T}^{G} 1_{T}$ for every irreducible representation $U$ of $G$. On the other hand, we see from the Plancherel formula for $G$ that any irreducible component of $\operatorname{Ind}_{T}^{G} 1_{T}$ is contained in $U \otimes U^{*}$ for an infinitely many inequivalent $U$ 's. In fact, consider the direct sum $R$ of $U \otimes U^{*}$ over a set of representatives of all equivalent classes of irreducible representations. Then, by the Peter-Weyl's theorem on the regular representation of $G, R$ is equivalent to a representation $M$ on $L^{2}(G, d g)$ given by

$$
\left(M\left(g_{0}\right) f\right)(g)=f\left(g_{0}^{-1} g g_{0}\right) \quad\left(f \in L^{2}(G, d g)\right),
$$

where $d g$ denotes the normalized Haar measure on $G$. Let us prove that $M$ is equivalent to an infinite multiple of $\operatorname{Ind}_{T}^{G} 1_{T}$. Every element $g$ in $G$ is expressed as

$$
g=k^{-1} t k \quad \text { with } k \in G, t \in T \text {. }
$$

Then the Weyl's integration formula says that for $f \in L^{1}(G, d g)$,

$$
\int_{G} f(g) d g=\frac{1}{|W|} \int_{T} \int_{T \backslash G} f\left(k^{-1} t k\right) d \bar{k}|\Delta(t)|^{2} d t
$$


where $d t$ and $d \bar{k}(\bar{k}=T k)$ denote the normalized invariant measures on $T$ and $T \backslash G$ respectively, $W$ is the Weyl group of $G$, and $\Delta(t)$ is a certain well-defined function on $T$. Let $G^{\prime}$ be the set of all regular elements of $G$, and $T_{1}$ a measurable section of $T^{\prime} / W$ in $T^{\prime}=T \cap G^{\prime}$. Then, the decomposition (2.11) of $g \in$ $G^{\prime}$ with $t \in T_{1}$ gives a measurable bijection of $G^{\prime}$ onto $(T \backslash G) \times T_{1}$ as $g \mapsto(\bar{k}, t)$, and by (2.12), $L^{2}(G, d g)$ is isomorphic to $\mathscr{H}=L^{2}\left(T_{1},|\Delta(t)|^{2} d t\right) \times L^{2}(T \backslash G, d \bar{k})$. The representation $M$ on the former is transformed to the representation $M_{1}$ on the latter: for $\varphi \times \psi \in \mathscr{H}$,

$$
M_{1}\left(g_{0}\right)(\varphi \times \psi)(t, \bar{k})=\varphi(t) \psi\left(\overline{k g}_{c}\right) .
$$

This proves our assertion.

\section{§3. Inequalities for multiplicities}

For an integral form $\omega \in\left(t_{c}\right)^{*}$, denote by $P_{K}(\omega)$ the number of different expressions of $\omega$ in a non-negative integral linear combination of positive roots. Then $P_{K}(\omega)$ is called the Kostant's partition function. In this section, we prove an inequality for multiplicities and, translating it by means of $P_{K}$, another one for the function $P_{K}$.

Let $\rho$ be half the sum of all positive roots of $\left(g_{c}, t_{c}\right)$. For a dominant integral form $\omega$, let $U_{\omega}$ be an irreducible representation of $G$ with highest weight $\omega$. For any weight $\theta$, denote by $m(\omega ; \theta)$ the multiplicity of $\theta$ in $U_{\omega}$. Then the Kostant's formula gives

$$
m(\omega ; \theta)=\sum_{w \in W} \operatorname{sgn}(w) P_{K}(w(\omega+\rho)-(\theta+\rho)),
$$

where $\operatorname{sgn}(w)$ denotes the usual sign of $w \in W$. Further let $\lambda, \mu$ and $\nu$ be dominant integral forms, and put

$$
m(\lambda, \mu, \nu)=\left[U_{\lambda} \otimes U_{\mu}: U_{\nu}\right] .
$$

Then we have the Steinberg's formula [1, Ch. VIII, § 9]

$$
m(\lambda, \mu, \nu)=\sum_{w, w^{\prime} \in W} \operatorname{sgn}\left(w w^{\prime}\right) P_{K}\left(w(\lambda+\rho)+w^{\prime}(\mu+\rho)-(\nu+2 \rho)\right) .
$$

Using Theorem 1, we get the following result.

THEOREM 4. Let $\lambda, \mu$ and $\nu$ be dominant integral forms. Then, $m(\lambda, \mu, \nu)$ $\leqslant m(\lambda ; \nu-\mu)$, or equivalently,

$$
-\sum_{\substack{w, w^{\prime} \in W \\ w^{\prime} \neq 1}} \operatorname{sgn}\left(w w^{\prime}\right) P_{K}\left(w(\lambda+\rho)+w^{\prime}(\mu+\rho)-(\nu+2 \rho)\right) \geqslant 0 .
$$

Proof. By Theorem 1, we have for dominant integral forms $\lambda_{1}, \lambda_{2}$ and $\lambda_{3}$, 


$$
\left[U_{\lambda_{1}} \otimes U_{\lambda_{2}}: U_{\lambda_{3}}\right] \leqslant\left[\operatorname{Ind}_{T}^{G} X_{\lambda_{1}+w_{0 \lambda_{2}}}: U_{\lambda_{3}}\right] \text {, }
$$

where $X_{\omega}$ denotes the character of $T$ given by $\omega \in\left(t_{c}\right)^{*}$. Applying the Frobenius reciprocity theorem on the right hand side, we get

$$
\left[U_{\lambda_{1}} \otimes U_{\lambda_{2}}: U_{\lambda_{3}}\right] \leqslant\left[U_{\lambda_{3}} \mid T: X_{\lambda_{1}+w_{0} \alpha_{2}}\right] .
$$

On the other hand, we have

$$
\left[U_{\lambda_{1}} \otimes U_{\lambda_{2}}: U_{\lambda_{3}}\right]=\left[U_{\lambda_{3}}^{*} \otimes U_{\lambda_{1}}: U_{\lambda_{2}}^{*}\right], \quad\left[U_{\lambda_{3}} \mid T: X_{\lambda_{1}+w_{02} 2}\right]=\left[U_{\lambda_{3}}^{*} \mid T: X_{-\lambda_{1}-w_{02} 2}\right] .
$$

The highest weight of $U_{i i}^{*}$ is equal to $\lambda_{i}^{*}=-w_{0} \lambda_{i}$. Therefore, putting $(\lambda, \mu, \nu)$ $=\left(\lambda_{3}^{*}, \lambda_{1}, \lambda_{2}^{*}\right)$, we get $m(\lambda, \mu, \nu) \leqslant m(\lambda ; \nu-\mu)$. Using (3.1), (3.2), this inequality is rewritten as (3.3).

Q.E.D.

Note 3. The value in (3.3) is equal to $m(\lambda ; \nu-\mu)-m(\lambda, \mu, \nu)$. In the case where $\mu=0$ and $\lambda \neq \nu, m(\lambda, \mu, \nu)=0$ and it gives a formula

$$
m(\lambda ; \nu)=-\sum_{w \in W, \neq 1} \operatorname{sgn}(w) m(\lambda ; \nu+\rho-w \rho) .
$$

This formula is well known [1, Ch. VIII, §9], and so is the inequality in this case.

\section{§4. Examples}

Let $G=U(n)$, the unitary group of order $n$, and $T$ the maximal torus of $G$ of all diagonal elements. For $\lambda \in t$, the subgroup $G(\lambda)$ is conjugate under $G$ to one of the following: for an ordered partition $\nu=\left(n_{1}, n_{2}, \cdots, n_{r}\right)$ of $n$, put

$$
H(\nu)=\left(\begin{array}{cccc}
U\left(n_{1}\right) & & & 0 \\
& U\left(n_{2}\right) & & \\
0 & & \ddots & \\
0 & & & U\left(n_{r}\right)
\end{array}\right) .
$$

Let $A=\left(m_{1}, m_{2}, \cdots, m_{n}\right)$ be an ordered set of integers. We define a function $A \Lambda$ on $T$ as follows. Let $\Im_{n}$ be the symmetric group of order $n$, and for $s=(s(1), s(2), \cdots, s(n)) \in \mathbb{S}_{n}$, put $s \Lambda=\left(m_{s(1)}, m_{s(2)}, \cdots, m_{s(n)}\right)$. Let $\delta=\operatorname{diag}\left(\delta_{1}\right.$, $\delta_{2}, \cdots, \delta_{n}$ ) be a diagonal matrix with diagonal elements $\delta_{1}, \delta_{2}, \cdots, \delta_{n}$. Then we put $\delta^{\Lambda}=\delta_{1}^{m_{1}} \delta_{2}^{m_{2}} \cdots \delta_{n}^{m_{n}}$, and

$$
A \Lambda=\sum_{s \in \Theta_{n}} \operatorname{sgn}(s) \delta^{s \Lambda},
$$

where $\operatorname{sgn}(s)$ denotes the sign of $s$. Put $\rho=(n-1, n-2, \cdots, 1,0)$, and assume 
$m_{1} \geqslant m_{2} \geqslant \cdots \geqslant m_{n}$ for $\Lambda$. Then the characters of irreducible representations of $G$ are given on $T$ by the formula

$$
\pi_{\Lambda}=A(\Lambda+\rho) / A \rho .
$$

Denote by $T(\Lambda)$ a representation with character $\pi_{\Lambda}$. The highest weight $\mu \epsilon$ $\left(\mathrm{t}_{c}\right) *$ of $T(\Lambda)$ is given by $\mu(X)=m_{1} x_{1}+m_{2} x_{2}+\cdots+m_{n} x_{n}$ for $X=\operatorname{diag}\left(x_{1}, x_{2}, \cdots\right.$, $\left.x_{n}\right) \in t_{c}$.

Let $H_{p}$ be $H(\nu)$ with $\nu=(1,1, \cdots, 1, n-p)$, i.e., $n_{1}=n_{2}=\cdots=n_{p}=1, n_{p+1}=$ $n-p$. Then we have

LEMma 4.1. A representation $T(\Lambda)$ of $G$ with parameter

$$
\Lambda=\left(m_{1}, m_{2}, \cdots, m_{n}\right), \quad m_{1} \geqslant m_{2} \geqslant \cdots \geqslant m_{n},
$$

is of class 1 with respect to $H_{p}$, that is, $\left[T(\Lambda): 1_{H_{p}}\right] \geqslant 1$, if and only if the following two conditions hold for $A$.

(1) $\sum_{1 \leqslant i \leqslant n} m_{i}=0$,

(2) the number of positive $m_{i}$ 's and that of negative $m_{i}$ 's are both $\leqslant p$.

Proof. The group $H_{1}$ is isomorphic to $U(1) \times U(n-1)$, and the restriction of $T(\Lambda)$ on $H_{1}$ is decomposed into irreducibles as

$$
\left.T(\Lambda)\right|_{H_{1}}=\sum T\left(\sum_{i=1}^{n} m_{i}-\sum_{j=1}^{n-1} m_{j}^{\prime}\right) \times T\left(m_{1}^{\prime}, m_{2}^{\prime}, \cdots, m_{n-1}^{\prime}\right),
$$

where $\left(m_{1}^{\prime}, m_{2}^{\prime}, \cdots, m_{n-1}^{\prime}\right)$ runs over all ordered set of integers satisfying

$$
m_{1} \geqslant m_{1}^{\prime} \geqslant m_{2} \geqslant m_{2}^{\prime} \geqslant m_{3} \geqslant \cdots \geqslant m_{n-1} \geqslant m_{n-1}^{\prime} \geqslant m_{n},
$$

and $T(m) \times T\left(m_{1}^{\prime}, m_{2}^{\prime}, \cdots, m_{n-1}^{\prime}\right)$ denotes a product of a representation $T(m)$ of $U(1)$ given by $U(1) \ni t \mapsto t^{m}$, and $T\left(m_{1}^{\prime}, m_{2}^{\prime}, \cdots, m_{n-1}^{\prime}\right)$ of $U(n-1)$. Using this fact inductively, we get the result.

Q.E.D.

ExAmple 1. Let $U$ be an irreducible representation of $G$ with highest weight $\mu$. Assume that $U \otimes U^{*} \subseteq \operatorname{Ind}_{H_{1}}^{G} 1_{H_{1}}$, then $G(\mu)$ contains a conjugate of $H_{1}$. This means that the conjecture is true in case $G(\lambda)=H_{1}$.

In fact, we know that $T(\Lambda) \subseteq \operatorname{Ind}_{H_{1}}^{G} 1_{H_{1}}$ if and only if the conditions (1), (2) hold for $\Lambda$. Now let $U=T(\Lambda)$. Then $U^{*}=T\left(\Lambda^{*}\right)$ with $\Lambda^{*}=\left(-m_{n},-m_{n-1}, \cdots\right.$, $\left.-m_{1}\right)$, whence $U \otimes U^{*}$ contains $T\left(\Lambda+\Lambda^{*}\right)$. Therefore, if $U \otimes U^{*} \varsigma_{\operatorname{Ind}_{H_{1}}}^{G} 1_{H_{1}}$, $\Lambda+\Lambda^{*}$ must satisfy the condition (2). This gives us $m_{2}-m_{n-1}=0$, and so $\Lambda$ is of the form

$$
\Lambda=(a+c, c, c, \cdots, c,-b+c), \quad a, b \geqslant 0 .
$$

To prove that $a=0$ or $b=0$, we apply Exercise 18) in [1, Ch. VIII, §7]. By 
this exercise, we see that if $a, b>0, T(\Lambda) \otimes T(\Lambda)^{*}$ contains $T\left(\Lambda^{\prime}\right)$ with $\Lambda^{\prime}=\Lambda+$ $\Lambda^{*}-(1,-1,0,0, \cdots, 0)=(a+b-1,1,0,0, \cdots, 0,-a-b)$. However $\Lambda^{\prime}$ does not satisfy the condition (2). This is a contradiction.

Note that when $a=0, G(\mu)$ contains a conjugate $H_{1}^{w_{0}}$ of $H_{1}$.

We know that the representation on the space of symmetric tensors of degree $q$ is $T\left(\Lambda^{q}\right)$ with $\Lambda^{q}=(q, 0,0, \cdots, 0)$, and that on the space of skew-symmetric tensors of degree $q$ is $T\left(\Lambda_{q}\right)$ with $\Lambda_{q}=(1,1, \cdots, 1,0, \cdots, 0)$ (the number of 1 is equal to $q$ ).

LEMMA 4.2.

(1) $T\left(\Lambda^{q}\right) \otimes T\left(\Lambda^{q}\right)^{*}=\sum_{r=0}^{q} T(r, 0,0, \cdots, 0,-r)$.

(2) $T\left(\Lambda_{q}\right) \otimes T\left(\Lambda_{q}\right)^{*}=\sum_{r=0}^{\min (q, n-q)} T(\underbrace{1,1, \cdots, 1}_{r}, 0, \cdots, 0,-\underbrace{1, \cdots,-1}_{r})$.

Proof. Here we prove (2). Note first that if we put for $\Lambda, \boldsymbol{S} \Lambda=\sum_{s \in \Phi_{n}} \delta^{s \Lambda}$, then

$$
S \Lambda \cdot A \Lambda^{\prime}=\sum_{s \in \subseteq_{n}} A\left(\Lambda^{\prime}+s \Lambda\right) .
$$

On the other hand, $\pi_{A_{q}}=A\left(\Lambda_{q}+\rho\right) / A \rho$, and also $\pi_{A_{q}}=N^{-1} S \Lambda_{q}$ with $N=\#\left\{s \in \widetilde{S}_{n}\right.$; $\left.s \Lambda_{q}=\Lambda_{q}\right\}$. Therefore the character of $T\left(\Lambda_{q}\right) \otimes T\left(\Lambda_{q}\right) *$ is given by

$$
\pi_{A_{q}} \cdot \bar{\pi}_{A_{q}}=N^{-1} A\left(\Lambda_{q}+\rho\right) S\left(\Lambda_{q}^{*}\right) / A \rho,
$$

where $\Lambda_{q}^{*}=(0,0, \cdots, 0,-1,-1, \cdots,-1)$. Then by (4.7),

$$
A\left(\Lambda_{q}+\rho\right) S\left(\Lambda_{q}^{*}\right)=\sum_{s \in \Subset_{n}} A\left(\Lambda_{q}+s \Lambda_{q}^{*}+\rho\right) .
$$

Picking up non-zero terms in the right hand side, we get the formula (2).

Q.E.D.

ExAMPle 2. From Lemmas 4.1 and 4.2 , we see that $T\left(\Lambda_{q}\right) \otimes T\left(\Lambda_{q}\right)^{*} \subseteq$ $\operatorname{Ind}_{H p}^{G} 1_{H_{p}}$ if and only if $\min (q, n-q) \leqslant p$. On the other hand, $\min (q, n-q) \leqslant$ $p$ if and only if $G(\mu)$ contains a conjugate of $H_{p}$, where $\mu$ denotes the highest weight of $T\left(\Lambda_{q}\right)$.

ADDED IN PROoF. Reexamining the proof of Theorem 2, we get the following amelioration of Theorem 3.

Theorem $3^{\prime}$. For $i=1,2$, let $\left(U_{i}, E_{i}, \mu_{i}\right)$ be as in Theorem 3, and let $v_{1} \in$ $E_{1}$ (resp. $v_{2} \in E_{2}$ ) be a non-zero highest (resp. lowest) weight vecior for $U_{1}$ (resp. for $U_{2}$ ). Then the vector $v=v_{1} \otimes v_{2}$ in $E_{1} \otimes E_{2}$ is a cyclic vector for $U_{1} \otimes U_{2}$, and satisfies that

$$
\left(U_{1} \otimes U_{2}\right)(h) v=\left(\chi_{\mu_{1}}\left(\chi_{\mu_{2}}\right)^{w_{0}}\right)(h) v \quad\left(h \in H=G\left(\mu_{1}\right) \cap G\left(\mu_{2}\right)^{w_{0}}\right) .
$$


Proof. Let $v_{i}^{*} \in E_{i}^{*}$ be a non-zero highest weight vector in $E_{i}^{*}$ for $U_{i}^{*}$. Then, in the proof of Theorem 2, the space $D$ is spanned by the functions $\varphi=$ $\varphi_{v^{\prime} v^{\prime \prime}}$ with $v^{\prime} \in E_{1}, v^{\prime \prime} \in E_{2}$, where

$$
\varphi_{v^{\prime} v^{\prime \prime}}\left(g_{1}, g_{2}\right)=\left(U_{1}\left(g_{1}\right) v^{\prime} \otimes U_{2}\left(g_{2}\right) v^{\prime \prime}, v_{1}^{*} \otimes v_{2}^{*}\right) \quad\left(g_{1}, g_{2} \in G_{c}\right) .
$$

The correspondence $\varphi \mapsto f$ of $D$ onto $\mathscr{E}$ induces a linear mapping of $E_{1} \otimes E_{2}$ onto $\mathscr{E}$ given by $v^{\prime} \otimes v^{\prime \prime} \mapsto f$, where

$$
f(g)=\left(U_{1}(g) v^{\prime} \otimes U_{2}\left(g_{w_{0}} g\right) v^{\prime \prime}, v_{1}^{*} \otimes v_{2}^{*}\right)=\left(v^{\prime} \otimes v^{\prime \prime},\left(U_{1} \otimes U_{2}\right)^{*}\left(g^{-1}\right) v^{*}\right)
$$

with $v^{*}=v_{1}^{*} \otimes U_{2}^{*}\left(g_{w_{0}}^{-1}\right) v_{2}^{*} \in E_{1}^{*} \otimes E_{2}^{*}$. The injectivity of the map corresponds to that the vector $v^{*}$ is cyclic for $\left(U_{1} \otimes U_{2}\right)^{*}$. Note that $U_{2}^{*}\left(g_{w_{0}}^{-1}\right) U_{2}^{*} \in E_{2}^{*}$ is a lowest weight vector for $U_{2}^{*}$. Then we get the assertion of the theorem for $U_{1}^{*}, U_{2}^{*}$.

Q.E.D.

We know also that Prof. N. Tatsuuma gave an algebraic proof of Theorem $3^{\prime}$ using the enveloping algebra of $\mathfrak{g}_{c}$.

\section{References}

[1] N. Bourbaki, Groupes et algèbres de Lie, Chap. 7 et 8, Hermann, 1975.

[2] C. Fronsdal, Some ideas about quantization, UCLA/77/TEP/18, October, 1977.

[3] S. Helgason, Differential geometry and symmetric spaces, Academic Press, 1962.

\section{FRONSDAL}

Physics Department, UCLA;

T. HIRAI

Department of Mathematics

KYOTO UNIVERSITY 